\title{
205 kA PULSE POWER SUPPLY FOR NEUTRINO FOCUSING HORNS
}

\author{
K. Bourkland, K. Roon, D. Tinsley \\ Fermilab, *P.O. Box 500, Batavia, IL 60510 \\ USA
}

\section{Abstract}

A new underground beamline is being constructed at Fermilab to generate and focus a beam of neutrinos on a detector 450 miles away in Soudan, Minnesota. A compact modulator utilizing capacitive energy storage and SCRs as the switching element has been built and tested at Fermilab. The $0.9 \mathrm{~F}$ capacitor bank operates at less than 1 $\mathrm{kV}$. It delivers its output of up to $240 \mathrm{kA}$ directly to the two series connected focusing horns via a multi-layer radiation hard stripline [1]. Dual pulse width capability allows for ready selection of $5.2 \mathrm{~ms}$, for slow beam spills, or $2.6 \mathrm{~ms}$ operation for reduced thermal stresses on the focusing horns during fast spill. Intended for installation in an underground equipment room, the design incorporates several novel features to facilitate transport, installation, and maintenance.

Various designs were examined to arrive at the most economical approach for providing the high pulse currents to the horns located in the very high radiation field, up to $3 \times 10^{\wedge} 7 \mathrm{kRads} / \mathrm{yr}$ absorbed dose of the beamline. These included charge recovery and electronic polarity reversal systems. The direct coupling approach was selected for its overall economy and compactness. The system has been operational for several months and results of those tests will be discussed. Controls and safety issues will also be discussed.

\section{INTRODUCTION}

Two focusing horns, each a single turn air core magnet constructed in a co-axial configuration, are located just downstream of a graphite beam target. They derive their name from the parabolic shape of the inner conductor. Figure 1 shows a cross-sectional view of the first of the two horns. Mounted in tandem in the beam, the two horns work together to form a two element magnetic lens. Their function is to focus the resultant particle shower following $120 \mathrm{GeV}$ protons on the upstream target. The physical spacing between horns can be selected depending upon the beam energy of interest, low, medium, or high. They require a power supply capable of providing 205kA pulses to achieve the desired 3 Tesla toroidal field between the inner and outer conductors. The pulses can be rectangular or $1 / 2$ sine wave, either would accomplish beam focusing.

*Fermilab is operated by Universities Research Assoc. Inc. under contract No. DE-AC02-76CH03000.
While a rectangular pulse would have the advantage of depositing less power into the horn, the system would have to operate at higher voltages. When operating with the horns in the low energy beam configuration, it is necessary that the target be installed 24" within the throat of the first horn. Of the two horns, horn 1 is located in the low-side of the system to keep the horn-to-target voltage to the lowest possible value, 65 volts and 40 volts for $2.6 \mathrm{~ms}$ and $5.2 \mathrm{~ms}$ pulse width operation respectively.

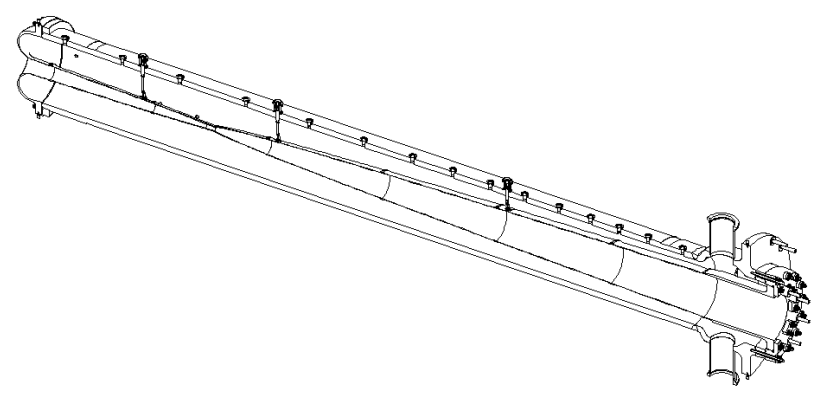

Figure 1. Horn 1, show in cross-section, is $13 " \varnothing \times 118$ " long. The center conductor wall thickness is 0.080 " at the ends, tapering gradually to 0.160 " in the neck region.

For slow beam-spill from Fermilab's Main Injector, a "flat-top" of $200 \mathrm{kA}$ for $1 \mathrm{~ms}$ is needed. A $5.2 \mathrm{~ms}, 1 / 2$ sine-wave pulse can provide this with a current variance of $\pm 2.5 \%$, operating between 195 and $205 \mathrm{kA}_{\mathrm{PK}}$. Current reading 'snapshots' captured during respective beam bunch passages can be used to generate correction factors for application to detector data, accommodating the current variance of the pulse. Fast beam spill can be accommodated with a much shorter current pulse to the horns, horn-to-target voltage permitting. . The $2.6 \mathrm{~ms}$ pulse raises horn-to-target voltages only modestly while significantly reducing both the electrical and mechanical stresses on the horns. A still narrower pulse width could suffice, but again at higher voltage. The charging source for the capacitor bank is an existing 12 pole phasecontrolled power supply recycled into this project from a decommissioned beamline application. Capacitor bank re-charging is accomplished between output pulses The system power dissipation is as show in Table 1 along with a tabulation of the various load elements.

Running at a $0.53 \mathrm{~Hz}$ rep-rate on a continuous basis and a requirement for a design life of ten years, the capacitor bank must operate for a minimum of 1.7E8 shots. 
Table 1. Beamline installation load elements

\begin{tabular}{|c|c|c|c|}
\hline System Component & $\underline{\mathrm{L}, \mu \mathrm{H}}$ & $\underline{\mathrm{R}, \mathrm{m} \Omega}$ & $\underline{\mathrm{P}}_{2.6} / \underline{\mathrm{P}}_{5.2}, \underline{\mathrm{kW}}$ \\
\hline Horn \#1 & 0.689 & 0.270 & $7.1 / 14.2$ \\
\hline Horn \#2 & 0.510 & 0.071 & $1.9 / 3.7$ \\
\hline \multicolumn{4}{|l|}{ Transmission line: $(16 \mathrm{nH}, 10 \mu \Omega / \mathrm{m})$} \\
\hline P. S. room to beamline, $9.5 \mathrm{~m}$. & 0.152 & 0.095 & $2.5 / 5.0$ \\
\hline Between horns, $53.5 \mathrm{~m}$. & 0.856 & 0.535 & $14.1 / 28.1$ \\
\hline Cap. Bank and internal bus & 0.1 & 0.050 & $1.3 / 2.6$ \\
\hline Stripline connections to horns & 0.1 & 0.010 & $0.26 / 0.52$ \\
\hline Total & $2.407 \mu \mathrm{H}$ & $1.031 \mathrm{~m} \Omega$ & $27.1 / 54.2 \mathrm{~kW}$ \\
\hline
\end{tabular}

\section{CONFIGURATION}

The configuration choices considered include a surface located HV P.S. with co-axial cables supplying primary power to beamline located step-down transformers and stripline to each horn, and similarly, cavern located HV P.S. with co-axial cables supplying primary power to stepdown transformers and stripline to each horn. With both of these proposals, the transformer(s) would have to be shielded from beamline radiation to achieve life expectancy. Transformers of this size exceeded $\$ 100 \mathrm{k}$ each plus a spare would be necessary. Since shielding was afforded by 30 feet of bedrock and power and utilities were already needed for other systems in the under-ground location, it was decided to locate the capacitor bank underground and direct couple it to the horns via stripline.

\section{DESIGN}

Due to the need for a low inductance transmission line and the high RMS current requirement, 8700 Arms, a planar design was chosen to connect the horns to the capacitor bank[1]. It utilizes air as the dielectric throughout its length, is spaced by zirconia ceramic insulators at 12" intervals and secured by bar clamps to complete the radiation hard design. Installation from capacitor bank to load is in a vertical orientation to allow for natural convection cooling. Stripline sections are coupled by the use of a spring style compression clamp. Contact areas are silver plated.

Since the stripline length is $<<1 / 4 \lambda$ at the operating frequencies, $100-200 \mathrm{~Hz}$, it is treated strictly as an inductance and resistance per unit length connected in series with the horn load. Previous measurements of the stripline configuration were made to establish values for $\mathrm{L}$ and $\mathrm{R}$ per unit length. By necessity, the stripline spacing increases in the regions where connections are needed and also where the breakouts to the horns occur. An inductance budget of $0.1 \mu \mathrm{H}$ was allowed for those connections. Likewise, an estimate was made for the capacitor bank internal inductance and was set at $0.1 \mu \mathrm{H}$. The stripline configuration is derived from a dual set of 12 " wide three layer, return-supply-return configuration where the supply layer is cleaved vertically to enhance cooling, as shown in Figure 2. Both aluminum and copper were considered for the stripline material. The skin depth at the operating frequencies are $8 \mathrm{~mm}$ and $6 \mathrm{~mm}$ respectively. For cost

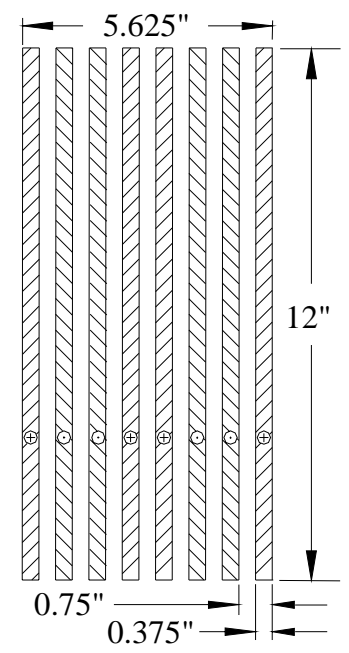

Figure 2. Stripline cross-section. and space considerations, conductor thickness was limited to one skin-depth. While copper has an advantage of lower resistance resulting in less energy loss, the added cost, weight, susceptibility to corrosion in the high humidity cavern environment, and longer cool-down time when activated by beam were disadvantages. The energy savings pay-back period for copper over aluminum was in excess of 40 years, four times longer than the projected life of the experiment. Aluminum electrical bus conductor, alloy 6101-T61, was the material of choice. It has nearly the conductivity of pure aluminum but with enhanced mechanical properties, short cool down time when activated by beam, and is readily available from commercial vendors in the desired dimensions.

\section{A. Capacitor Bank}

Segmented metalized polypropylene film capacitors built by NWL to Fermilab specification are used in this application. Utilizing 5.8 micron film, they are rated at 670 working volts and specified to hi-pot to $1,340 \mathrm{Vdc}$, a safety margin of two. The total bank capacitance of $0.9 \mathrm{~F}$ is comprised of 120 units of $7.5 \mathrm{mF}, \pm 5 \%$ tolerance. Space is provided for two additional capacitors per cell to accommodate potential future load parameter changes. The two pulse widths, $5.2 \mathrm{~ms}$ or $2.6 \mathrm{~ms}$, are obtainable by connecting all units in parallel, or by paralleling 60 series pairs, respectively. An interchangeable set of 'jumpers' facilitates conversion in a few hours time.

A SPICE circuit analysis of the overall power supply and load indicated that less than $12 \%$ of the initial energy would be recoverable after each discharge cycle. 


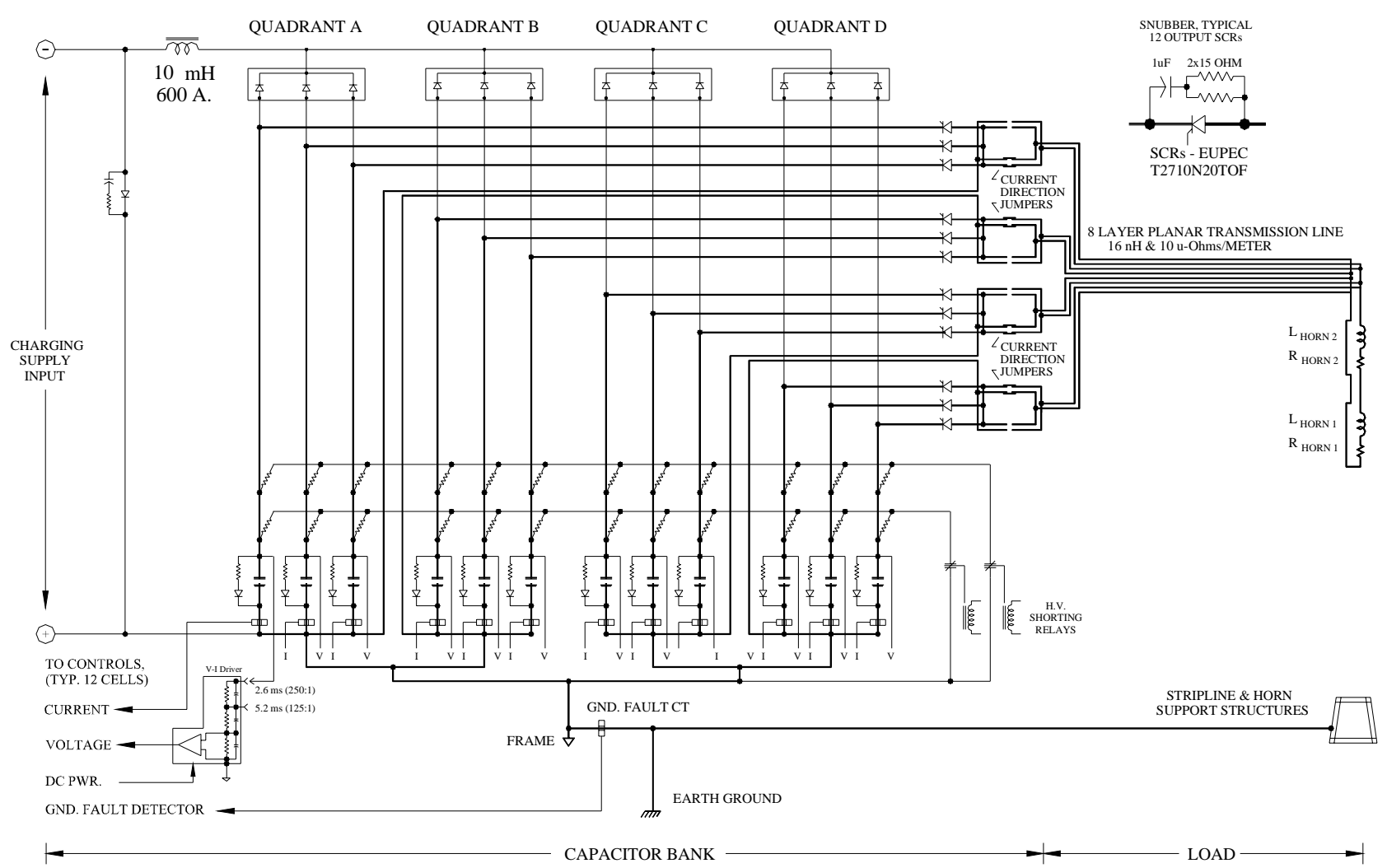

Figure 3. Schematic of capacitor bank, stripline and horn load.

Concepts initially considered for energy recovery included the use of a separate reactor (large), cycling recovery current through the load after beam passage (lossy), and a concept whereby the capacitor bank was electrically reversed after each pulse by use of charging source and load reversing SCR arrays, eliminating the need for a recovery reactor. With little energy to be recovered, the added complexity was judged not to be cost effective. Subsequently, a freewheeling diode in series with a resistor is installed across each capacitor cell to dissipate the reflected energy prior to recharging the bank for the next pulse.

With the operating current for the horns specified at 205 $\mathrm{kA}$, a design margin of $20 \%$ is added to establish an engineering design value of $240 \mathrm{kA}$ for the capacitor bank and SCR switch. Experience has shown that the 20\% margin is sufficient to accommodate possible subsequent modifications to beamline optics as well as variations necessitated by beamline tuning, without adding undue cost to the construction.

Candidate SCRs were run in a R-L-C test circuit at 25 $\mathrm{kA}_{\mathrm{PK}}$ while duplicating operating values of voltage, pulse width, and cooling for a minimum of 10E6 shots, based upon an initial decision to use just eight capacitor cells and SCRs.

For safety, the capacitor bank is divided into 12 cells of $1 / 12$ the total capacitance each, keeping the amount of stored energy per cell at $\sim 50 \%$ of the energy containment rating of the capacitor cases. To limit energy delivered to a possible capacitor fault, the cells are isolated from each other and the charging source by diodes, and isolated from each other and the load by SCRs. With only one SCR needed per cell, current sharing is also assured. The final design uses 12 Eupec T2710N20TOF devices sharing the total current, each device operating at $17 \mathrm{kA}$ PK. Three cells, forming a 'quadrant,' drive a stripline pair through their respective SCRs. Each of the quadrants drive one-forth of the stripline.

For reduced stress on the horns it is desirable to keep stripline currents balanced to $\leq 1 \%$. To achieve this, the stripline, shown in Figure 4, is routed into the geometric center of the capacitor bank with symmetry maintained in all three dimensional axis. Additionally, the total capacitance in each cell, nominally $75 \mathrm{mF}$, was initially matched within $2 \mathrm{uF}$ by careful selection through the range of in-tolerance values to obtain 12 matched sets.

Provision is made to reverse the direction of the output current of the capacitor bank via mechanical means by exchanging 'jumper' links in the stripline internal to the enclosure. While this could have been accomplished electrically by additional SCRs, the need was seen as infrequent and the expense not justifiable.

Each cell of the capacitor bank is monitored by a separate frequency compensated voltage divider that includes an active voltage-to-current converter to provide noise immunity plus the ability to drive long cables. A current transformer is installed in each cell for reading current output. System operation voltages are 860 Volts and 515 Volts for the $2.6 \mathrm{~ms}$ and $5.2 \mathrm{~ms}$ pulse widths respectively. 


\section{B. Controls}

Operation of the power supply system is monitored by low-level electronic controls. Each of the 12 voltage signals is compared to the average of the 12 cell voltages on a continuous basis throughout the charge-discharge cycle. Similarly, the 12 current signals are averaged and compared on a continuous basis. Window detectors set to detect variations greater than $\pm 2.5 \%$ of the average will terminate system operation. The CT signals of the three cells of each quadrant are added to provide a current readout for each stripline pair. All $12 \mathrm{CT}$ signals are added together to provide a total load current readout and provide for over-current monitoring.

To prevent any circulating currents in the structure, the capacitor bank is grounded only to a single point of the enclosure. This locale is the connection point for two 930 $\mathrm{mcm}$ bonding cables, one going to each horn shield (mounting) module, and separately and earth ground. They are monitored by a CT connected into the controls interlock system to terminate system operation in the event of a loadto-ground fault.

\section{Structure}

The system was built and is being operated for horn testing in an assembly building on the surface while civil construction of the beamline cavern is still underway. The $22,000 \mathrm{lb}$. capacitor bank will ultimately be transported to the underground beamline utility room at a depth of 130 '

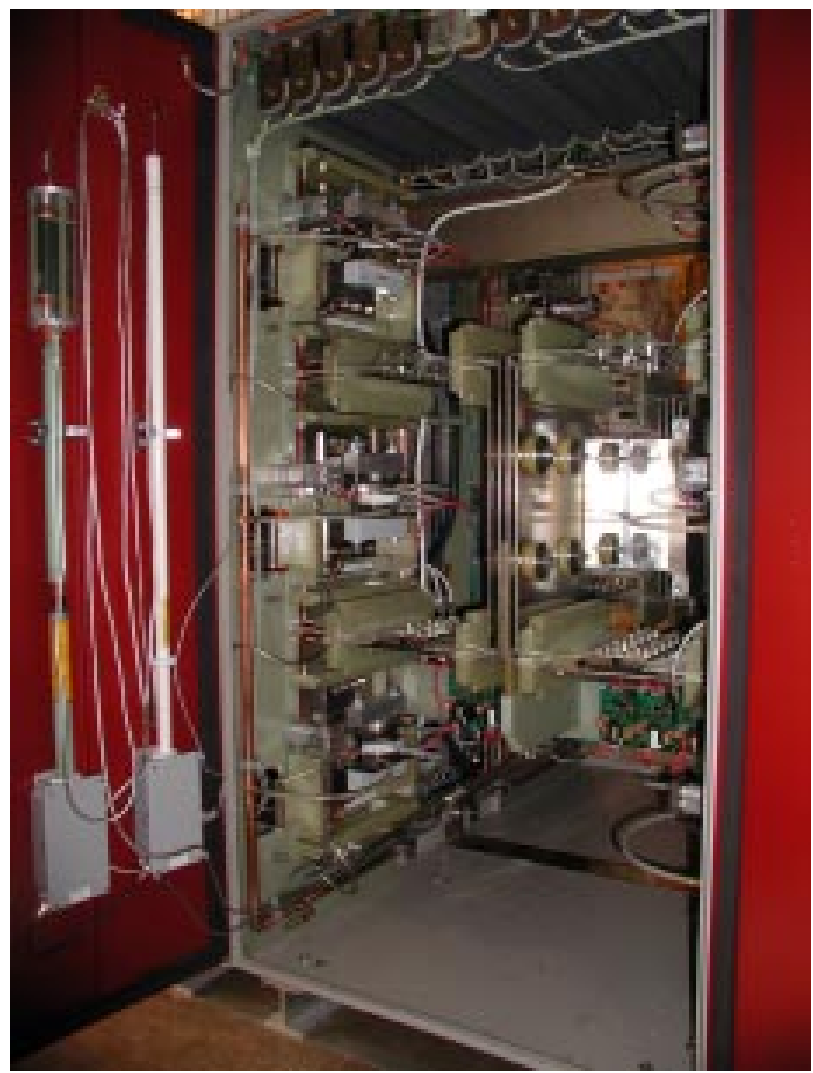

Figure 4. Center bay of enclosure showing the stripline routing and SCR installation of one quadrant.

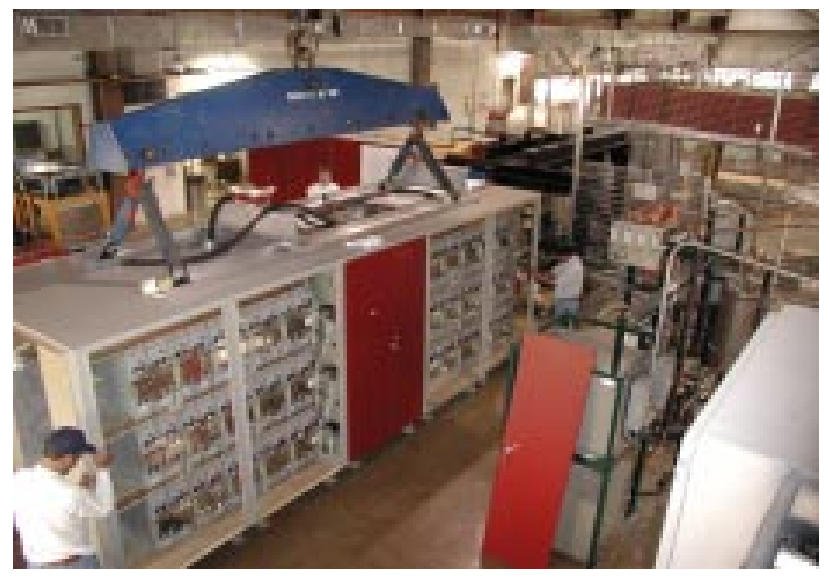

Figure 5. Capacitor bank being lifted and transported.

through a 22' diameter vertical access shaft as a completed and tested unit without disassembly. Since the capacitors alone constitute $50 \%$ of the total weight, the intent of the mechanical design was to lift this weight directly while being transported. This is accomplished by making the capacitor support shelves of structural aluminum and aluminum plate. The four capacitor shelf assemblies, one in each corner of the enclosure, are then braced internally to each other with aluminum I-beam. The balance of the internal component weight is carried through the enclosure structure to the shelf assemblies. The entire unit, Figure 5, is lifted via four load- rated lifting eyes attached to the shelf structures through the top of the enclosure.

\section{PERFORMANCE}

The pulse-to-pulse monitoring controls were sensitive enough to detect early-on one capacitor whose bushing nuts were not completely tightened, and one of six bolts in a stripline connection not tightened to the correct torque value, resulting in minor arching. Once corrected, these areas have not been a recurring problem. To date, the capacitor bank, stripline, and horn load have operated for 6E6 pulses without incident or failure. Current balance between the four stripline pairs is within $0.2 \%$. During operation, the peak sound level was measured to be 125 $\mathrm{db}$ (a) during the pulse. Vibration studies of the horn and stripline indicated that stripline vibration is being coupled into the horn, but is not seen as a serious concern. We continue to be vigilant for metal fatigue related failures than can occur, particularly in the high current bus, as a consequence of the repetitive pulsed magnetic forces. Another area being closely monitored are the stripline connections. To date they have been problem free.

\section{REFERENCES}

[1] K. Bourkland, C. Jensen, D. Tinsely, W. Markel, "High Current Pulse Striplines," IEEE Pulse Power / Plasma Science Conference, June 2001. 
